\title{
Poly(4-vinylphenol) coated magnetic nanoparticles based dispersive solid-phase microextraction of the determination of mercury(II) in water
}

\author{
Benrabha A.M.F. and Tay K.S.* \\ Department of Chemistry, Faculty of Science, University of Malaya, 50603 Kuala Lumpur, Malaysia \\ Received: 06/08/2021, Accepted: 26/09/2021, Available online: 04/10/2021 \\ *to whom all correspondence should be addressed: e-mail: khengsoo@um.edu.my \\ https://doi.org/10.30955/gnj.003901
}

\section{Graphical abstract}

Magnetic Dispersive Solid Phase Extraction of $\mathrm{Hg}^{2+}$ using poly(4-vinylphenol) coated magnetic particles
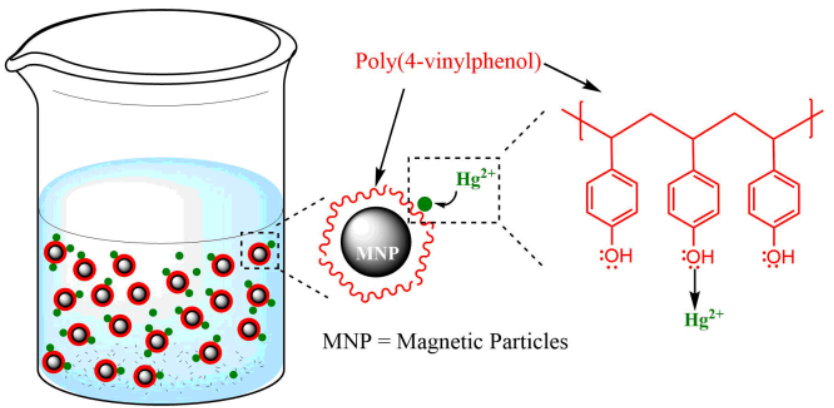

MNP $=$ Magnetic Particles

\section{Abstract}

The development of magnetic sorbent for dispersive solidphase micro-extraction (DmSPE) often requires lengthy multi-step reactions. This research revealed a simplified method for preparing magnetic sorbent for the DmSPE using poly(4-vinylphenol) (PVP). The magnetic sorbent (PVP@MNP) was prepared by coating PVP on magnetic particles (MNP). The characterization and formation of PVP@MNP were confirmed using infrared spectroscopy, scanning electron microscope, and energy-dispersive $\mathrm{X}$ ray spectroscopy. The primary goal of this study is to develop a sensitive DmSPE method to analyze $\mathrm{Hg}^{2+}$ in water using PVP@MNP as a magnetic sorbent. The preparation of PVP@MNP was performed in a simple coating method at room temperature. Briefly, the PVP@MNP was prepared by sonicating the mixture of MNP and PVP. This sorbent was then used as a magnetic sorbent for the extraction of $\mathrm{Hg}^{2+}$ from water. The developed PVP@MNP based DmSPE reached a low method of detection limit $\left(0.01 \mu \mathrm{g} \mathrm{L}^{-1}\right)$ and limit of quantification $\left(0.04 \mu \mathrm{g} \mathrm{L}^{-1}\right)$. This method also showed a wide linearity range $\left(100-2000 \mu \mathrm{g} \mathrm{L}^{-1}\right)$ with a good correlation factor under optimized conditions. The developed method showed good recovery (72-90\%) with good intraday and interday precision. This study also showed that the developed DmSPE method was effectively used to determine $\mathrm{Hg}^{2+}$ in drinking water, mineral water, and surface water. The result also demonstrated that PVP@MNP is reusable.

Keywords: mercury (II) ion, magnetic particles, adsorption, removal mechanism, metal extraction, preconcentration.

\section{Introduction}

The presence of heavy metals in water has become one of the most challenging environmental concerns (Shirani et al., 2020). Although most of the heavy metals occurred in the water at trace level, it has been found to cause a toxic effect on living organisms due to its bioaccumulative behavior (Imran et al., 2021; Marrugo-Negrete et al., 2021). Among heavy metals, mercury $(\mathrm{Hg})$ is known as toxic metal, which has been found to cause a wide range of adverse effects on the living organism (Dibbern et al., 2021). Hg pollution has remained a major health concern. Therefore, rapid detection and analysis of $\mathrm{Hg}^{2+}$ ions at trace levels are in critical need to protect those who are vulnerable (Pokhrel et al., 2017).

Recently, dispersive micro solid-phase extraction (DmSPE) has gained much attention in sample preparation techniques because of its simplicity, speed, and efficiency (Chisvert et al., 2019). This miniaturized sample preparation method involved the dispersion of a specific solid-phase or so-called sorbent in water matrices to extract targeted analytes. After extraction, the sorbent is often isolated via filtration or centrifugation. Then, the targeted analytes can be eluted from the sorbent using a specific reagent or solvent. The use of magnetic particles as sorbent has further simplified the DmSPE in sorbent isolation and elution. The magnetic sorbent can be isolated from water matrices using a magnet, and in this case, centrifugation is not required. The technique for the isolation of magnetic sorbent is more economical than the conventional sorbent without magnetic properties.

Bare magnetic $\mathrm{Fe}_{3} \mathrm{O}_{4}$ is not suitable for being used as sorbent due to the poor surface properties to adsorbed most of the analyte and easily oxidized characteristic. 
Consequently, research in this area has focused on developing new magnetic sorbents to enhance their efficiency, selectivity, physical and chemical stability (Chisvert et al., 2019). Surface modification of magnetic particles with a ligand or specific chemical to enhance the adsorption of metal ions is one of the common methods to produce magnetic sorbent for DmSPE (Alinezhad et al., 2020; Chen et al., 2021; Ma et al., 2020). This method often involves lengthy multi-step reactions, such as coating of magnetic particles, modifying the coated magnetic particles with a specific functional group or chemical, and the attached ligand (Fu et al., 2017). In this study, a simplified method was used to synthesize the poly(4-vinylphenol) (PVP) coated magnetic particles (PVP@MNP). PVP@MNP was used as a sorbent of DmSPE for the determination of $\mathrm{Hg}^{2+}$ in water. The application of PVP exhibits advantages that include insoluble in water, rapid and strong complexation with metals, low-cost and non-toxic nature (Zhang et al., 2017). The coating of polymer on the magnetic particles could also enhance its stability during DmSPE by avoiding direct contact with the atmosphere. The objectives of this study were (1) to synthesize the PVP@MNP, (2) to study the behavior of PVP@MNP in removing $\mathrm{Hg}^{2+}$ from water, and (3) to develop a PVP@MNP based DmSPE method for the determination of $\mathrm{Hg}^{2+}$ in water. According to the literature search, the application of PVP@MNP as sorbent for DmSPE has not been reported.

\section{Materials and methods}

\subsection{Materials}

Iron (II) chloride tetrahydrate, ferric chloride hexahydrate, ammonium hydroxide solution (25\%), HPLC grade isopropanol, sodium dihydrogen phosphate monohydrate, disodium hydrogen phosphate, and mercury standard solution (1000 ppm) were purchased from Merck (Germany). Poly(4-vinylphenol) was obtained from Sigma Aldrich. Ultrapure water was produced by using an Elga water purification system (UK). The Neodymium disc magnet (20 mm diameter $\times 3 \mathrm{~mm}$ height) was purchased from Eclipse Magnetics (UK). Drinking water and mineral water were purchased at a local market.

\subsection{Synthesis of the MNP and PVP coated magnetic particles (PVP@MNP)}

Magnetic particle (MNP) was synthesized using the coprecipitation method at room temperature in the air (Khor et al., 2019). Briefly, iron (II) chloride tetrahydrate and ferric chloride hexahydrate with a molar ratio of 2:3 were first dissolved in $50 \mathrm{~mL}$ deionized water in a beaker. Then $50 \mathrm{~mL}$ of ammonium hydroxide solution (25\%) was added under stirring. The mixture was then stirred vigorously for $30 \mathrm{~min}$ at room temperature. The obtained MNP was isolated using an external magnetic field, washed thoroughly with deionized water, and then vacuum dried.

For the synthesis of PVP@MNP, $0.5 \mathrm{~g}$ of MNP and $0.5 \mathrm{~g}$ of Poly(4-vinylphenol) (Sigma Aldrich) were added into the $50 \mathrm{~mL}$ isopropanol (Merck) in a conical flask. The mixture was sonicated for $30 \mathrm{~min}$. The PVP@MNP were isolated from the isopropanol by using a neodymium magnet. The obtained PVP@MNP were rinsed thoroughly with deionized water and isopropanol. PVP@MNP was dried under vacuum and kept in a desiccator before being used.

\subsection{Instrumentation for PVP@MNP characterization}

Perkin Elmer Spectrum 400 ATR-FTIR spectrophotometer was used to record the Fourier-transform infrared (FTIR) spectra. The resolution of $2 \mathrm{~cm}^{-1}$ and 16 scans were applied to obtain the FTIR spectrum. The Scanning Electron Microscope equipped with Energy Dispersive X-ray (EDX) spectroscopy (Hitachi, SU8200) was used to capture the morphology of the MNP and PVP@MNP and determined the elemental composition.

\subsection{Adsorption and kinetics studies}

To obtain an optimized method, the amount of adsorbent, adsorption time, the $\mathrm{pH}$ of the solution, and the initial concentration of $\mathrm{Hg}^{2+}$, were optimized using one variable at a time method. The effect of operating parameters was evaluated by monitoring the percentage removal of $\mathrm{Hg}^{2+}$. All experiments were performed in triplicate. The removal of $\mathrm{Hg}^{2+}$ by PVP@MNP was carried out in a $30 \mathrm{ml}$ polypropylene bottle. For the adsorption study, $10 \mathrm{mg}$ of PVP@MNP was added to the $20 \mathrm{~mL}$ of $1 \mathrm{mg} \mathrm{L}^{-1} \mathrm{Hg}^{2+}$. The mixture was shaken for the defined time interval. Then, the PVP@MNP was separated from the solution using the neodymium magnet. The remaining solution was sampled, and the concentration of $\mathrm{Hg}^{2+}$ was determined using $\mathrm{a}$ Mercury Analyzer (NIC RA-3).

The isolated adsorbent was washed with deionized water and vacuum dried. The isolated PVP@MNP was dispersed into a $0.5 \mathrm{~mL}$ of desorption reagent and shaken using the orbital shaker for $5 \mathrm{~min}$ for the $\mathrm{Hg}^{2+}$ desorption. PVP@MNP was separated from the desorption reagent using a neodymium magnet placed at the outer part of the vial. The amount of $\mathrm{Hg}^{2+}$ in the desorption reagent was determined using Mercury Analyzer. The $\mathrm{Hg}^{2+}$ recovery calculated according to Equation (1):

$$
\text { Recovery }=\frac{C_{\mathrm{ex}}}{C_{0}} \times 100 \%
$$

where $C_{\text {ex }}$ represents the concentration of extracted $\mathrm{Hg}^{2+}$ and $C_{0}$ refers to the initial concentration of $\mathrm{Hg}^{2+}$ used for the extraction.

\subsection{Determination of the performance of PVP@MNP based DISPE in real sample analysis}

The optimized PVP@MNP based method was used to determine $\mathrm{Hg}^{2+}$ in drinking water, mineral water, and surface water. The surface water was collected from the lake around Kuala Lumpur, Malaysia. The selected water samples were filtered through the $0.45 \mu \mathrm{m}$ membrane filter. These water samples were free from $\mathrm{Hg}^{2+}$. Consequently, these water samples were spiked with the known concentration of $\mathrm{Hg}^{2+} . \mathrm{Hg}^{2+}$ in water samples was pre-concentrated with the optimized DmSPE conditions: $\mathrm{pH}$ 7, 10 mg of PVP@MNP, 60 min agitation for extraction, $0.5 \mathrm{~mL}$ of desorption reagent $(2 \%$ thiourea in $0.1 \mathrm{M} \mathrm{HCl}$ (TUA-HCl)), and 5 min of agitation for desorption. 


\section{Results and discussion}

\subsection{PVP@MNP characterization}

(a)

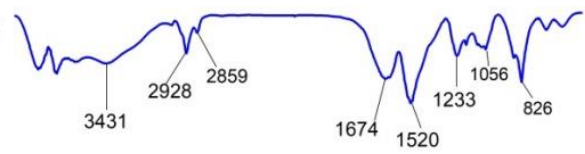

(b)
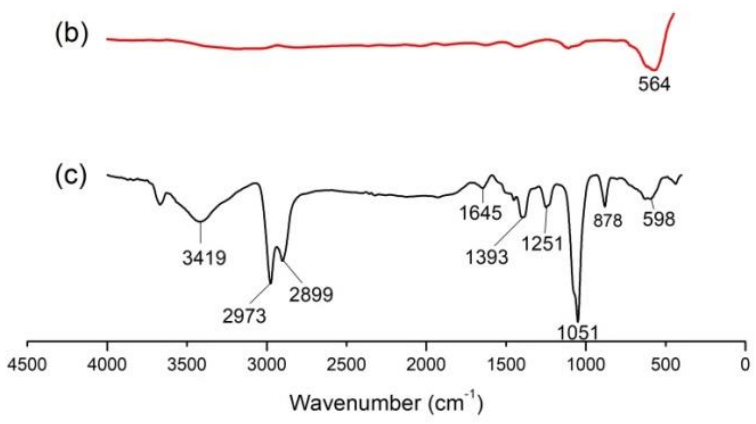

Figure 1. FTIR spectra of (a) PVP, (b) MNP and, (c) PVP@MNP.

FTIR analysis was carried out to confirm the presence of PVP on MNP after the coating process. Figure 1 shows the FT-IR spectra of MNP, PVP, and PVP@MNP. FTIR spectrum of MNP showed a significant peak at $564 \mathrm{~cm}^{-1}$, indicating the presence of Fe-O (Lesiak et al., 2019). PVP shows the peaks at 3431 and $2800-2900 \mathrm{~cm}^{-1}$ were due to $\mathrm{OH}$ stretching and $\mathrm{C}-\mathrm{H}$ stretching of the aromatic ring, respectively (Zhang et al., 2017). PVP also shows a peak at $1674 \mathrm{~cm}^{-1}$ due to the $\mathrm{C}-\mathrm{H}$ bending of the aromatic ring. The peak at $1233 \mathrm{~cm}^{-1}$ indicates the presence of the $\mathrm{C}-\mathrm{O}$ bond in PVP. The emergence of the peaks from both PVP $\left(3419,2973,2899,1645 \mathrm{~cm}^{-1}\right)$ and $\operatorname{MNP}\left(598 \mathrm{~cm}^{-1}\right)$ in PVP@MNP suggesting PVP was successfully coated on MNP. To further confirm this result, elemental analysis was performed on the MNP and PVP@MNP. The PVP@MNP showed the presence of $22.2 \%$ of carbon. In contrast, carbon was not detected in MNP (Table 1). This result further indicated that the PVP@MNP was successfully synthesized. Figure 2 shows the SEM image of MNP and PVP@MNP. There are no significant changes in the morphology after coating. Both MNP and PVP@MNP showed spherical shapes. The size of the PVP@MNP was ranged from 11-23 $\mathrm{nm}$, which is slightly larger than MNP with the size ranging from $7-15 \mathrm{~nm}$. This result could be useful to further indicate the presence of PVP on the MNP.

Table 1. Elemental composition of MNP and PVP@MNP

\begin{tabular}{ccc}
\hline \multirow{2}{*}{ Element } & \multicolumn{2}{c}{ Percentage (\%) } \\
\cline { 2 - 3 } & MNP & PVP@MNP \\
\hline $\mathrm{Fe}$ & 60.3 & 26.6 \\
\hline $\mathrm{O}$ & 39.7 & 51.2 \\
\hline $\mathrm{C}$ & 0 & 22.2 \\
\hline
\end{tabular}

\subsection{Optimization of PVP@MNP based DmSPE}

In general, the amount of sorbent plays a huge influence in the adsorption of analytes in the DmSPE process. The sorbent dosage determines the surface area and the number of active sites for the adsorption (Ghodsi et al.,
2021). In order to obtain the maximum adsorption efficiency, the impact of the amount of PVP@MNP was investigated in the range of 10 to $40 \mathrm{mg}$. the percent removal was slightly increased from 82.3 to $96.8 \%$ when the amount of PVP@MNP was increased from 10 to $40 \mathrm{mg}$ (Figure 3a). However, this increment was found to be insignificant. Therefore, for further studies, $10 \mathrm{mg}$ of PVP@MNP was selected to reduce sorbent usage. The dispersion of the PVP@MNP was performed using an orbital shaker at room temperature. Consequently, the agitation time was investigated to ensure the PVP@MNP was dispersed effectively in the water for achieving the most efficient $\mathrm{Hg}^{2+}$ adsorption. The percent removal of $\mathrm{Hg}^{2+}$ was increased significantly to $93.4 \%$ at the $10 \mathrm{~min}$ of adsorption time (Figure $3 \mathrm{~b}$ ). The percent $\mathrm{Hg}^{2+}$ removal was increased to $95.9 \%$ at $30 \mathrm{~min}$ of agitation time. The result also showed that the percentage of $\mathrm{Hg}^{2+}$ removal was further increased to $98.6 \%$ at $60 \mathrm{~min}$, and no significant improvement of $\mathrm{Hg}^{2+}$ removal was observed after $60 \mathrm{~min}$. Consequently, $60 \mathrm{~min}$ of agitation time was chosen for further study to improve the adsorption efficiency of $\mathrm{Hg}^{2+}$ by PVP@MNP.
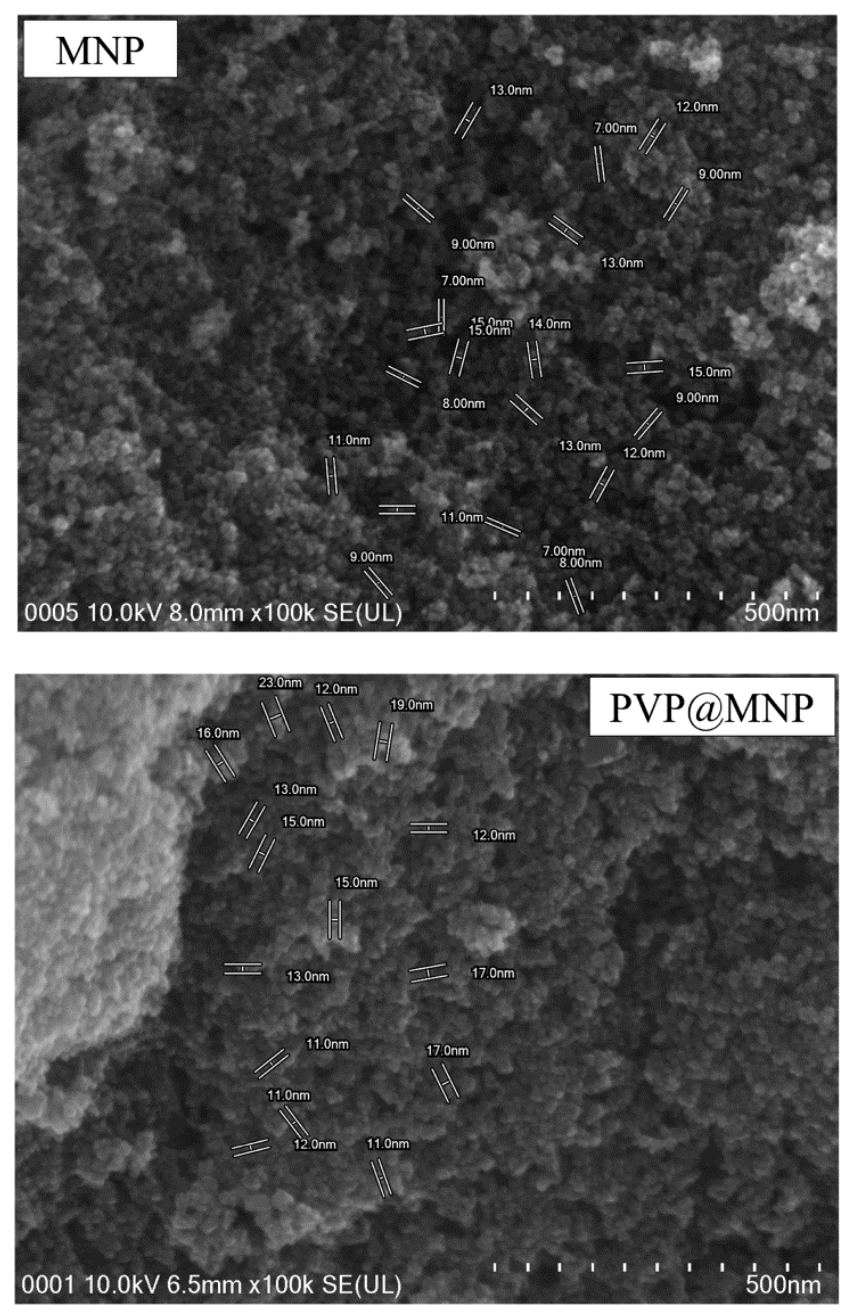

Figure 2. Scanning electron microscope image of MNP and PVP@MNP.

The $\mathrm{pH}$ of the solution influences the speciation of $\mathrm{Hg}^{2+}$ in water and the degree of ionization of PVP. To study the effect of $\mathrm{pH}$ on the $\mathrm{Hg}^{2+}$ removal, the $\mathrm{pH}$ of the solution 
was varied from 3 to 9. The percent removal of $\mathrm{Hg}^{2+}$ by PVP@MNP was 5.75\% at pH 3. However, the percentage removal was increased significantly to $99.9 \%$ when the $\mathrm{pH}$ was varied from 4.0 to 9.0 (Figure $3 \mathrm{c}$ ). At $\mathrm{pH} \mathrm{3,} \mathrm{the} \mathrm{low}$ removal efficiency of $\mathrm{Hg}^{2+}$ was because of the competition between $\mathrm{H}^{+}$with $\mathrm{Hg}^{2+}$ and $\mathrm{Hg}(\mathrm{OH})^{+}$for the active sites of PVP@MNP (Abdallah et al., 2020). When the pH was increased to $\mathrm{pH} 4$, the phenol group of PVP was ionized to form negatively charged phenolate, which could interact favorably with $\mathrm{Hg}^{2+}$ via ionic interaction. As reported by Abdallah et al. (2020), water-soluble $\mathrm{Hg}(\mathrm{OH})_{2}$ is the main species at $\mathrm{pH} 5$ and above. The high removal efficiency of $\mathrm{Hg}^{2+}$ at high $\mathrm{pH}$ indicated that $\mathrm{Hg}(\mathrm{OH})_{2}$ interacted favorably with PVP@MNP. At $\mathrm{pH} 7$, the $\mathrm{Hg}^{2+}$ removal was found to achieve $99.9 \%$. Therefore, this $\mathrm{pH}$ condition was selected for the following experiment. The selection of $\mathrm{pH}$ 7 is also an advantage for environmental analysis because $\mathrm{pH}$ adjustment is not required. (a)

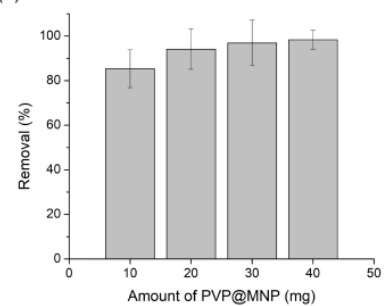

(c)

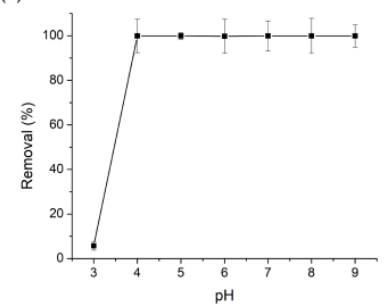

(e)

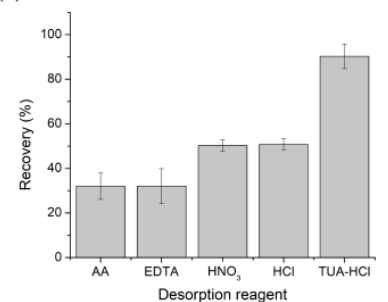

(b)

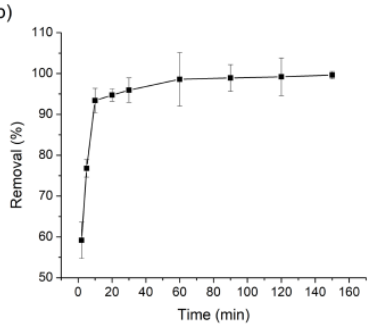

(d)

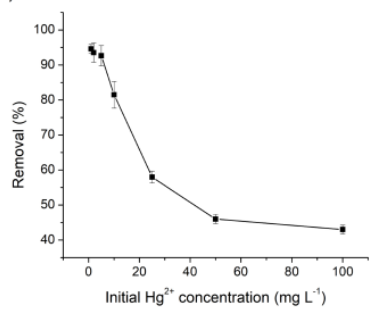

(f)

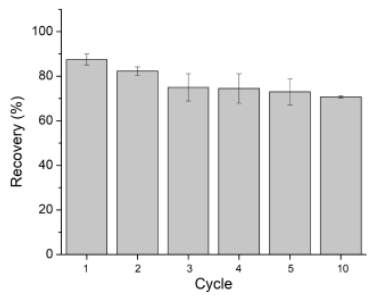

Figure 3. The influence of (a) the amount of PVP@MNPs $\left(\left[\mathrm{Hg}^{2+}\right]=1 \mathrm{mg} \mathrm{L}^{-1}\right.$, volume of $\mathrm{Hg}^{2+}$ solution $=20 \mathrm{~mL}, \mathrm{pH}=7$ and adsorption time $=60 \mathrm{~min}$ ), (b) adsorption time (Amount of adsorbent $=10 \mathrm{mg},\left[\mathrm{Hg}^{2+}\right]=1 \mathrm{mg} \mathrm{L}^{-1}, \mathrm{pH}=7$ and volume of $\mathrm{Hg}^{2+}$ solution $=20 \mathrm{~mL}$ ), (c) pH (Amount of adsorbent $=10 \mathrm{mg}$, $\left[\mathrm{Hg}^{2+}\right]=1 \mathrm{mg} \mathrm{L}^{-1}$, volume of aqueous solution $=20 \mathrm{~mL}$ and adsorption time $=60 \mathrm{~min}$ ), (d) $\mathrm{Hg}^{2+}$ concentration (Amount of adsorbent $=10 \mathrm{mg}$, volume of aqueous solution $=20 \mathrm{~mL}$, adsorption time $=60 \mathrm{~min}$ and $\mathrm{pH}$ of aqueous solution $=7$ ) on the removal of $\mathrm{Hg}^{2+}$ in aqueous sample, (e) different desorption reagents on the recovery of $\mathrm{Hg}^{2+}$ from PVP@MNP and (f) Reusability of PVP@MNP.

The experiment for the effect of $\mathrm{Hg}^{2+}$ concentration showed that when the concentration of $\mathrm{Hg}^{2+}$ was increased from 0.5 to $10 \mathrm{mg} \mathrm{L}^{-1}$, the percentage of $\mathrm{Hg}^{2+}$ removal by PVP@MNP was decreased from 97.0 to $81.5 \%$
(Figure 3d). The effectiveness of $\mathrm{Hg}^{2+}$ adsorption was found to diminish further from 81.5 to $43.0 \%$ with increasing concentration of $\mathrm{Hg}^{2+}$ from 10 to $100 \mathrm{mg} \mathrm{L}^{-1}$. This observation was mainly due to the occurrence of a higher amount of $\mathrm{Hg}^{2+}$ in the solution when the concentration of $\mathrm{Hg}^{2+}$ increased. However, the available active sites for the adsorption of $\mathrm{Hg}^{2+}$ remained constant when the amount of PVP@MNP was fixed (Al-Ghouti et al., 2019).

For searching the most effective reagent to desorb the $\mathrm{Hg}^{2+}$ from PVP@MNP, the performance of $0.1 \mathrm{M}$ acetic acid $\left(\mathrm{CH}_{3} \mathrm{COOH}\right), 0.1 \mathrm{M}$ ethylenediaminetetraacetic acid (EDTA), $0.1 \mathrm{M}$ nitric acid $\left(\mathrm{HNO}_{3}\right), 0.1 \mathrm{M}$ hydrochloric acid $(\mathrm{HCl})$, and $2 \%$ thiourea in $0.1 \mathrm{M} \mathrm{HCl}$ solution (TUA-HCl) were evaluated. These reagents have the ability to solubilize metal ions (Alguacil et al., 2020). The desorption process was carried out by agitating the PVP@MNP loaded with $\mathrm{Hg}^{2+}$ with $0.5 \mathrm{~mL}$ of the selected desorption reagents. According to Figure $3 e, T U A-H C l$ shows the highest efficiency in extracting $\mathrm{Hg}^{2+}$ from PVP@MNP.TUA$\mathrm{HCl}$ is usually utilized to desorb $\mathrm{Hg}^{2+}$ ions from sorbents because the sulfur and nitrogen atoms in thiourea have a strong affinity to complex with $\mathrm{Hg}^{2+}$. Also, chloride ions from $\mathrm{HCl}$ could complex with the $\mathrm{Hg}^{2+}$ and result in the formation of $\mathrm{HgCl}_{2}$ (Velempini et al., 2019).

The reusability of a sorbent is a significant factor to consider when deciding on the sustainability of analytical method (Fu et al., 2017). Ten cycles of adsorptiondesorption were carried out continuously on a single adsorbent sample, and the results are shown in Figure $3 \mathrm{f}$. Considering the $\mathrm{Hg}^{2+}$ in the real environment is far lower than 1 ppm, PVP@MNP could be reused. PVP@MNP for ten consecutive adsorption cycles (Alinezhad et al., 2020). The adsorption property of PVP@MNP showed only a slightly decreased after ten cycles of adsorptiondesorption 87.5 to $70.0 \%$ (Figure $3 f$ ).

\subsection{Mechanism of the adsorption}

The adsorption data were fitted to Langmuir and Freundlich isotherm models to understand how $\mathrm{Hg}^{2+}$ is adsorbed by PVP@MNP (Naushad et al., 2019). Langmuir model describes the monolayer adsorption of sorbate by homogenous active sites of the sorbent (Bushra et al., 2019). The linearized form of the Langmuir model can be written as:

$$
\frac{C_{\mathrm{e}}}{Q_{\mathrm{e}}}=\frac{1}{Q_{\mathrm{m}} B_{\mathrm{L}}}+\frac{C_{\mathrm{e}}}{Q_{\mathrm{m}}}
$$

where $C_{e}$ is the concentration of $\mathrm{Hg}^{2+}$ in water after reaching the equilibrium, $Q_{e}$ represents the equilibrium adsorption capacity. $Q_{m}$ is the maximum adsorption capacity, and $B_{\mathrm{L}}$ is the Langmuir constant related to the energy of adsorption. $Q_{e}$ was calculated using Equation (3):

$$
Q_{e}=\left(\frac{C_{o}-C_{e}}{m}\right) v
$$

where $C_{o}$ is the initial concentration of $\mathrm{Hg}^{2+}, m$ represents the mass of PVP@MNP, and $V$ is the volume of the $\mathrm{Hg}^{2+}$ 
solution. On the other hand, the Freundlich model describes the multilayer adsorption of sorbate on nonuniform active sites of the sorbent with a heterogeneous surface (Bushra et al., 2019). The linearized form of the Freundlich model is given in Equation (4):

$$
\log Q_{e}=\log B_{F}+\frac{1}{n} \log C_{e}
$$

where $B_{F}$ is the Freundlich constant related to adsorption capacity, and $n$ represents the adsorption intensity constant. The coefficient of determination $\left(R^{2}\right)$ value of the Freundlich model was 0.975, which was higher than the Langmuir model with the $\mathrm{R}^{2}$ value of 0.838 (Figures $4 a$ and b). This observation recommended that PVP@MNP adsorbed the $\mathrm{Hg}^{2+}$ via a multilayer adsorption process described by the Freundlich model. Table 2 shows the adsorption isotherm parameters for Langmuir and Freundlich model. The separation factor, $R_{L}$, was calculated to determine the favorability of the adsorption of $\mathrm{Hg}^{2+}$ by PVP@MNP using Equation 5. The adsorption process is defined as favorable if $R_{L}$ values are fall within 0 to 1 . The adsorption process is unfavorable or linear if values are larger than 1 or equal to 0 , respectively. The $R_{L}$ values obtained for this study was ranged from 0.08-0.89 (Table 2), which indicated that the adsorption process of $\mathrm{Hg}^{2+}$ by PVP@MNP is favorable.

$$
R_{L}=\frac{1}{1+\left(B_{L} \times C_{0}\right)}
$$

As mentioned in the previous section, the adsorption of $\mathrm{Hg}^{2+}$ by PVP@MNP is expected to proceed via complexation. Consequently, the data obtained were fitted to the pseudo-first-order and pseudo-second-order kinetic models. Pseudo-first-order kinetic model describes the physical adsorption that the adsorption rate is influenced by the number of available adsorption sites on the materials (Shojaeiarani et al., 2021). On the other hand, the pseudo-second-order model indicates the chemisorption between sorbate and adsorbent, including the exchange or transfer of valence electrons (Ho, 2006). The linearized form of pseudo-first-order and pseudosecond-order models can be described by Equation (6) and (7):

$$
\begin{aligned}
& \log \left(Q_{e}-Q_{t}\right)=\log Q_{e}-\frac{k}{2.303} t \\
& \frac{t}{Q_{t}}=\frac{t}{Q_{e}}+\frac{1}{K Q_{e}^{2}}
\end{aligned}
$$

where $Q_{t}$ is the amount of adsorbed sorbate at time $t . k$ is the rate constant of the pseudo-first-order, and $K$ is the pseudo-second-order rate constant. Figures $4 \mathrm{c}$ and $\mathrm{d}$ show that the data were well fitted to the pseudo-secondorder with $\mathrm{R}^{2}$ near to 1 . The $\mathrm{R}^{2}$ value of pseudo-first-order was 0.9238 , which is lower than the pseudo-second-order. This result showed the adsorption of $\mathrm{Hg}^{2+}$ by PVP@MNP was mainly happened through chemisorption.
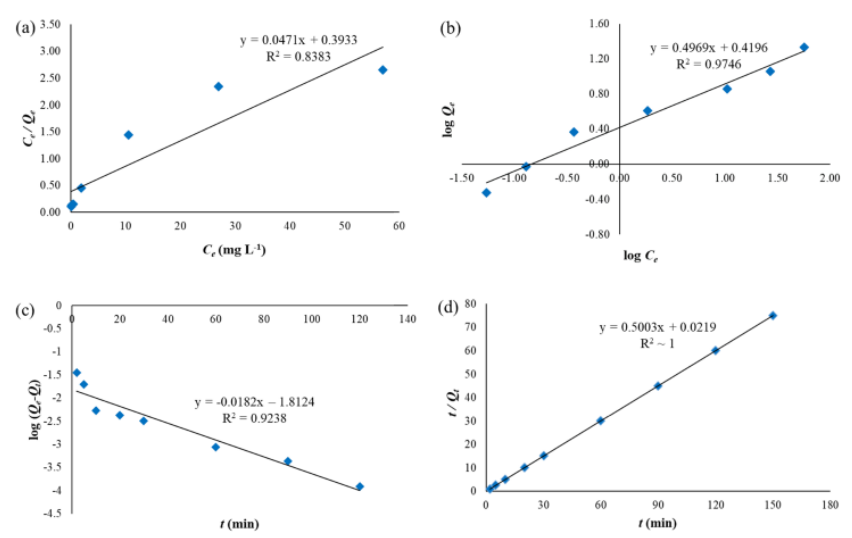

Figure 4. (a) Adsorption isotherm Langmuir model, (b) adsorption isotherm Freundlich model, and (c) Pseudo-secondorder model for adsorption of $\mathrm{Hg}^{2+}$ on PVP@MNP.

Table 2 Adsorption isotherm parameters of Langmuir and Freundlich model

\begin{tabular}{ccccc}
\hline Adsorption Isotherm model & \multicolumn{2}{c}{ Parameter } & $\boldsymbol{R}_{\mathrm{L}}$ & $\boldsymbol{R}^{\mathbf{2}}$ \\
\hline Langmuir & $\boldsymbol{Q}_{\mathrm{m}}(\mathrm{mg} / \mathrm{g})$ & $\boldsymbol{B}_{\mathrm{L}}(\mathrm{L} / \mathrm{mg})$ & $0.08-0.89$ & 0.8383 \\
\hline Freundlich & 21.1 & 0.1199 & $\boldsymbol{n}$ & $\boldsymbol{R}^{\mathbf{2}}$ \\
\hline & $\boldsymbol{B}_{\mathrm{F}}(\mathrm{L} / \mathrm{mg})$ & $\mathbf{1 / n}$ & $\mathbf{2 . 0 1}$ & 0.9746 \\
\hline
\end{tabular}

\subsection{Analytical performance of PVP@MNP based DmSPE in water analysis}

The analytical performance of the optimized PVP@MNP based DmSPE method was assessed using linearity, the method detection limit (MDL), the limit of quantitation (LOQ), enrichment factor, inter-day, and intra-day precision. The enrichment factor (EF) was determined using the sample spiked with $\mathrm{Hg}^{2+}$ at $1000 \mu \mathrm{g} \mathrm{L}^{-1}$. EF value was obtained using the following equation:

$$
E F=\frac{C_{F}}{C_{0}}
$$

where $C_{0}$ and $C_{F}$ refer to the initial and final concentration of $\mathrm{Hg}^{2+}$. The calculated $\mathrm{EF}$ value was 40 . The optimized method showed a good correlation factor $\mathrm{R}^{2}$ near to 1 for the linear range of 100 to $2000 \mu \mathrm{g} \mathrm{L}^{-1}$. In this experiment, MDL and LOQ were determined by analyzing seven replicates of deionized water spiked with $\mathrm{Hg}^{2+}$ at the concentration equal to signal to noise ratio of 5 . MDL and LOQ were calculated using Equations (7) and (8):

$$
\begin{aligned}
& M D L=S \times(t \text {-value }) \\
& L O Q=S \times 10
\end{aligned}
$$

where $S$ is the standard deviation of the concentration of $\mathrm{Hg}^{2+}$ obtained from the seven replicates of analysis. The $t-$ value of 3.143 , representing $99 \%$ of a confidence level, was used to calculate the MDL (Ripp, 1996). The obtained 
MDL and LOQ for the optimized method were 0.01 and $0.04 \mu \mathrm{g} \mathrm{L}^{-1}$, respectively.

The intraday and interday reproducibility of the PVP@MNP based DmSPE was expressed in percent relative standard deviation (\%RSD) by analyzing sets of four and five replicates, respectively. The \%RSD obtained were ranged from 3.4-7.3\% for intraday reproducibility and $1-5.1 \%$ for interday reproducibility. The analytical performance of the developed PVP@MNP based DmSPE was comparable with other reported DmSPE techniques in terms of detection limit and LOQ (Table 3). The developed method was also achieved lower detection limits than most of the reported studies. Therefore, it can be concluded that the developed method was an effective procedure for the preconcentration of $\mathrm{Hg}^{2+}$ in water.

For validation, drinking water, mineral water, and surface water spiked with $\mathrm{Hg}^{2+}$ at the concentration of 100,500 , and $2000 \mu \mathrm{g} \mathrm{L}^{-1}$ were analyzed using the optimized method. The intraday and interday recovery and precision of the optimized method are presented in Table 4. The obtained recovery for $\mathrm{Hg}^{2+}$ was ranged from 72 to $90 \%$, with the relative standard deviation of $1-5 \%$ for both the intra-day and inter-day precision, respectively. These results indicated that the matrices of the selected water samples do not significantly impact the performance of the developed method.

Table 3. Comparison of the developed method with other DmSPE methods in the $\mathrm{Hg}^{2+}$ analysis

\begin{tabular}{|c|c|c|c|c|}
\hline \multicolumn{2}{|c|}{ Absorbent } & Detection limit $\left(\mu \mathrm{gL}^{-1}\right)$ & $\operatorname{LOQ}\left(\mu \mathrm{gL}^{-1}\right)$ & Reference \\
\hline \multicolumn{2}{|c|}{ Dendrimer functionalized magnetic nanosorbent } & 0.03 & Not reported & Ghodsi et al., 2021 \\
\hline \multicolumn{2}{|c|}{ Ion-imprinted magnetic nanoparticle } & 0.084 & 0.28 & Jiang et al., 2017 \\
\hline \multicolumn{2}{|c|}{ 1,5-Diphenylcarbazide functionalized magnetic sorbent } & 0.16 & Not reported & Zhai et al., 2010 \\
\hline \multicolumn{2}{|c|}{ PVP@MNP } & 0.01 & 0.04 & This study \\
\hline \multicolumn{5}{|c|}{ Table 4 Recoveries of $\mathrm{Hg}^{2+}$ in the real water samples } \\
\hline \multirow{2}{*}{ Sample } & \multirow{2}{*}{ Spiked $\left[\mathrm{Hg}^{2+}\right]\left(\mu \mathrm{L} \mathrm{L}^{-1}\right)$} & \multicolumn{3}{|c|}{ *RSD\% } \\
\hline & & Inter-day $(n=4)$ & & Intra-day (n=5) \\
\hline \multirow{4}{*}{ Drinking water } & 0 & ND & & ND \\
\hline & 100 & $75 \pm 1$ & & $76 \pm 2$ \\
\hline & 500 & $77 \pm 1$ & & $76 \pm 1$ \\
\hline & 2000 & $83 \pm 3$ & & $84 \pm 4$ \\
\hline \multirow{4}{*}{ Mineral water } & 0 & ND & & ND \\
\hline & 100 & $75 \pm 2$ & & $78 \pm 1$ \\
\hline & 500 & $79 \pm 1$ & & $79 \pm 1$ \\
\hline & 2000 & $78 \pm 1$ & & $90 \pm 5$ \\
\hline \multirow{4}{*}{ Surface water } & 0 & ND & & ND \\
\hline & 100 & $72 \pm 1$ & & $85 \pm 1$ \\
\hline & 500 & $75 \pm 5$ & & $89 \pm 2$ \\
\hline & 2000 & $86 \pm 1$ & & $80 \pm 5$ \\
\hline
\end{tabular}

* Experiments were carried out within 22-26 March 2021

\section{Conclusion}

In this study, a magnetic adsorbent, PVP@MNP, was synthesized through a single-step coating method at room temperature. The PVP@MNP was then used as a sorbent to develop a DmSPE method for the analysis of $\mathrm{Hg}^{2+}$ in water. The developed PVP@MNP based DmSPE showed a low method detection limit at $0.01 \mathrm{\mu gL}^{-1}$ and LOQ at 0.04 $\mu \mathrm{gL}^{-1}$. This method also showed a wide range of linearity with good repeatability. The PVP@MNP was suitable for repeated use over ten cycles without significant loss in the initial adsorption capacity. This method also showed that the determination of $\mathrm{Hg}^{2+}$ in drinking water, mineral water, and surface water reached a recovery efficiency of 72 to $90 \%$ with low \%RSD for intraday and interday reproducibility. In conclusion, this performance of the developed PVP@MNP based DmSPE method is comparable with other reported DmSPE methods.

\section{Acknowledgments}

This research was supported financially by the Ministry of Higher Education Malaysia (Fundamental Research Grant Scheme No. FP042-2016).

\section{References}

Abdallah S.A., Tay K.S., Low K.H. (2020). Feasibility of mercury (II) ion removal by nitrated polycarbonate derived from waste optical discs. International Journal of Environmental Science and Technology, 17, 4161-4170.

Al-Ghouti M.A., Da'ana D., Abu-Dieyeh M., Khraisheh M. (2019). Adsorptive removal of mercury from water by adsorbents derived from date pits. Scientific Report, 9, 15327.

Alguacil F.J., López F.A. (2020). Adsorption processing for the removal of toxic $\mathrm{Hg}$ (II) from liquid effluents: Advances in the 2019 year. Metals, 10, 412.

Alinezhad H., Zabihi M., Kahfroushan D. (2020). Design and fabrication the novel polymeric magnetic boehmite nanocomposite (boehmite@ $\mathrm{Fe}_{3} \mathrm{O}_{4} @ \mathrm{PLA} @ \mathrm{SiO}_{2}$ ) for the remarkable competitive adsorption of methylene blue and mercury ions. Journal of Physics and Chemistry of Solids, 144, 109515.

Bushra R., Ahmed A., Shahadat M. (2017). Mechanism of adsorption on nanomaterials, In: Advanced Environmental Analysis: Applications of Nanomaterials, Hussain C.M. and Kharisov B. (Eds.), The Royal Society of Chemistry: London, UK. 
Chen Y., He M., Chen B., Hu B. (2021). Thiol-grafted magnetic polymer for preconcentration of $\mathrm{Cd}, \mathrm{Hg}$, $\mathrm{Pb}$ from environmental water followed by inductively coupled plasma mass spectrometry detection. Spectrochimica Acta Part B: Atomic Spectroscopy, 177, 106071.

Chisvert A., Cárdenas S., Lucena R. (2019). Dispersive micro-solid phase extraction. Trends in Analytical Chemistry, 112, 226233.

Dibbern M., Elmeros M., Dietz R., Søndergaard J., Michelsen A., Sonne C. (2021). Mercury exposure and risk assessment for Eurasian otters (Lutra lutra) in Denmark. Chemosphere, 272, 129608.

Fu Y., Chen Z., Ying S., Wang J., Hu J. (2017) Functionalized magnetic mesoporous silica/poly(m-aminothiophenol) nanocomposite for $\mathrm{Hg}(\mathrm{II})$ rapid uptake and high catalytic activity of spent $\mathrm{Hg}(\mathrm{II})$ adsorbent. Science of the Total Environment, 574, 1379-1388.

Ghodsi S., Behbahani M., Badi M.Y., Ghambarian M., Sobhi H.R., Esrafili A. (2021). A new dendrimer-functionalized magnetic nanosorbent for the efficient adsorption and subsequent trace measurement of $\mathrm{Hg}$ (II) ions in wastewater samples. Journal of Molecular Liquids, 323, 114472.

Ho Y. (2006). Review of second-order models for adsorption systems. Journal of Hazardous Materials, 136, 681-689.

Imran U., Mahar R.B., Ullah A. Shaikh K. (2021). Seasonal Variability of Heavy Metals in Manchar Lake of Arid Southern Pakistan and Its Consequential Human Health Risk. Polish Journal of Environmental Studies, 30, 163-175.

Jiang W., Jin X., Yu X., Wu W., Xu L., Fu F. (2017). Ion-imprinted magnetic nanoparticles for specific separation and concentration of ultra-trace methyl mercury from aqueous sample. Journal of Chromatography A, 1496, 167-173.

Khor S.W., Lee Y.K., Tay K.S. (2019). Selective magnetic mercury(II) ion capturing ligand-doped silica gel for water analysis. Analyst, 144, 1968-1974.

Lesiak B., Rangam N., Jiricek P., Gordeev I., Tóth J., Kövér L., Mohai M., Borowicz P. (2019) Surface study of $\mathrm{Fe}_{3} \mathrm{O}_{4}$ nanoparticles functionalized with biocompatible adsorbed molecules. Frontiers in Chemistry, 7, 642.

Ma J., Wang H., Zhang M., Li D., Liu L., Yang H. (2020). Preparation of terpyridine-functionalized paramagnetic nickel-zinc ferrite microspheres for adsorbing $\mathrm{Pb}(\mathrm{ii}), \mathrm{Hg}$ (ii), and Cd(ii) from water. RSC Advances, 10, 39468.

Marrugo-Negrete J., Pinedo-Hernández J., Marrugo-Madrid S., Díez S. (2021). Assessment of trace element pollution and ecological risks in a river basin impacted by mining in Colombia. Environmental Science and Pollution Research, 28, 201-210.

Naushad M., Ahamad T., AlOthman Z.A., Al-Muhtaseb A.H. (2019). Green and eco-friendly nanocomposite for the removal of toxic $\mathrm{Hg}$ (II) metal ion from aqueous environment: Adsorption kinetics \& isotherm modeling. Journal of Molecular Liquids, 279, 1-8.

Pokhrel L.R., Ettorre N., Jacobs Z.L., Zarr A., Weir M.H., Scheuerman P.R., Kanel S.R., Dubey B. (2017). Novel carbon nanotube (CNT)-based ultrasensitive sensors for trace mercury(II) detection in water: A review. Science of the Total Environment, 574, 1379-1388.

Ripp J. (1996). Analytical detection limit guidance \& laboratory guide for determining method detection limits, Wisconsin
Department of Natural Resources: Laboratory Certification Program.

Shirani M., Afzali K.N., Jahan S., Strezov V., Soleimani-Sardo M. (2020). Pollution and contamination assessment of heavy metals in the sediments of Jazmurian playa in southeast Iran. Scientific Report, 10, 4775.

Shojaeiarani J., Shirzadifar A., Bajwa D.S. (2021) Robust and porous 3D-printed multifunctional hydrogels for efficient removal of cationic and anionic dyes from aqueous solution. Microporous and Mesoporous Materials, 327, 111382.

Velempini T., Pillay K., Mbianda X.Y., Arotiba O.A. (2019). Carboxymethyl cellulose thiol-imprinted polymers: Synthesis, characterization and selective $\mathrm{Hg}(\mathrm{II})$ adsorption. Journal of Environmental Sciences, 79, 280-296.

Zhai Y., He Q., Yang X., Han Q. (2010). Solid phase extraction and preconcentration of trace mercury (II) from aqueous solution using magnetic nanoparticles doped with 1,5diphenylcarbazide. Microchimica Acta, 169, 353-360.

Zhang G., Kim C., Choi W. (2017). Poly(4-vinylphenol) as a new stable and metal-free sensitizer of titania for visible light photocatalysis through ligand-to-metal charge transfer process. Catalysis Today, 281, 109-116. 